\title{
Recognition of Outliers by Sampling Ends
}

National Cancer Institute

\section{Source}

National Cancer Institute. Recognition of Outliers by Sampling Ends. NCI Thesaurus.

Code C121991.

A hierarchical clustering method for the unsupervised, unbiased selection of gene expression patterns. The ROSE probe set clusters are based on the size of the outlier group they identify as well as the magnitude of the deviation from expected intensity. 